\title{
Light Touch Power Supply Switch Using For Portable Device
}

\author{
Zhao Liangliang $^{1,2}$, Hao Shaojie ${ }^{1,2}$, Sun Jiawen ${ }^{1,2}$, Zhang Feng ${ }^{1,2}$, Zhou Hao ${ }^{1,2}$, Han Junhui ${ }^{1,2}$ \\ 1.Science and Technology on Electronic Test \& Measurement Laboratory \\ 2. The 41st Research Institute of CETC \\ Qingdao, China
}

Keywords: Portable equipment; Light touch; Microprocessor; Power supply switch.

\begin{abstract}
With the development of science and technology, modern electronic products are becoming more and more lightweight, and tend to miniaturization. Switch the equipment also put forward higher requirements, the use of light touch switch to replace the traditional mechanical switch has become a trend, that can prolong the life of the switch, effectively reduce to improve the stability of the power system. This paper introduces several light touch type power switch used by experimental verification. The circuit has the advantages of simple structure, small occupied space, working stably and widely used in portable devices.
\end{abstract}

\section{Introduction}

With the development of science and technology, especially the rapid development of microelectronics technology, more and more portable and handheld devices are gradually replacing the cumbersome instruments in many fields, but also it opens up more and more direction. If the mechanical switch is used, not only the life span is short, but also the reliability of the system will be affected. According to market survey, the micro switch with simple and easy feature is popular in the market. ${ }^{1}$ Light touch switch and straight key switch sales best in market. Most manufacturers shift to product touch switch, which even becomes the trend. Whether civil, such as audio, microwave oven, lampblack machine, or the military, such as individual combat equipment, there are specific requirements for portable equipment. Light touch type power supply switch will be applied widely.

\section{The basic principle of light touch switch}

The power switch generally has a bistable characteristic. The bistable characteristic is necessary to control the power supply through light touch switch. Light touch switch cannot maintain a steady state, so other circuits must be needed. As is shown in Figure 1, principle diagram of power supply light touch switch includes the touch switch hardware, electronic switch, load, power supply etc. According to the two states of the switch hardware to control the electronic switch, the whole switch system maintains two steady states, to achieve the control of the power supply switch. According to different requirements, light touch switch can provide some additional features, such as automatically turned on, the delay off, state indication. More additional features can be achieved if microprocessor is used. ${ }^{2}$

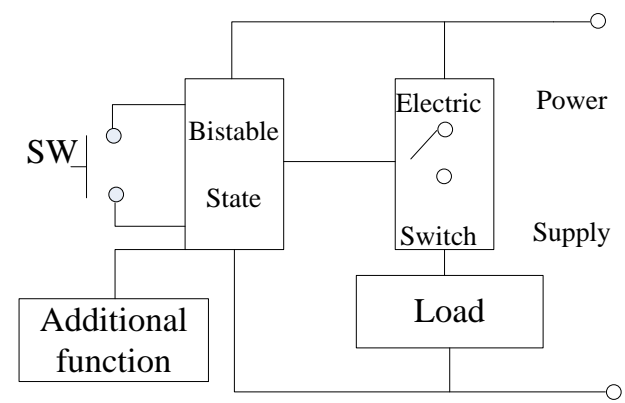

Fig.1: The principle diagram of light touch switch

\section{Cases of light touch power supply switch}

According to the engineering experience, several validated light touch power supply switch circuit will be introduced, are validated using. Readers can choose one or more to improve according to their own needs.

\subsection{Light touch power supply switch based on transistors}

The transistor has the advantages of simple structure and small space occupation. If in the absence of additional functional requirements, light touch power switch based on transistors should be the first choice of touch switch. Light touch power supply switch has a common problem that cannot close itself power, leakage current and increase power consumption. ${ }^{3}$ The circuit structure in this paper can effectively solve this problem.

As is shown in figure 2, two transistors, one is type PNP and the other is type NPN. The working principle is similar to that of Silicon controlled rectifier. On power up, because the voltage on the C2 can't burst, so the Q2 tube cut-off. Q1 tube because there is no base current. And the output voltage Vout is zero. The Q2 tube maintains a cut off state due to a lack of base current. The input voltage through R1, R2 and D1 charge on the $\mathrm{C} 1$, so that the voltage on the $\mathrm{C} 1$ will close to the input 
power supply voltage. After charging, all the circuits are in the cut-off state, in addition to the weak leakage current, load and switch are not power consumption, the switch is in cut off state.

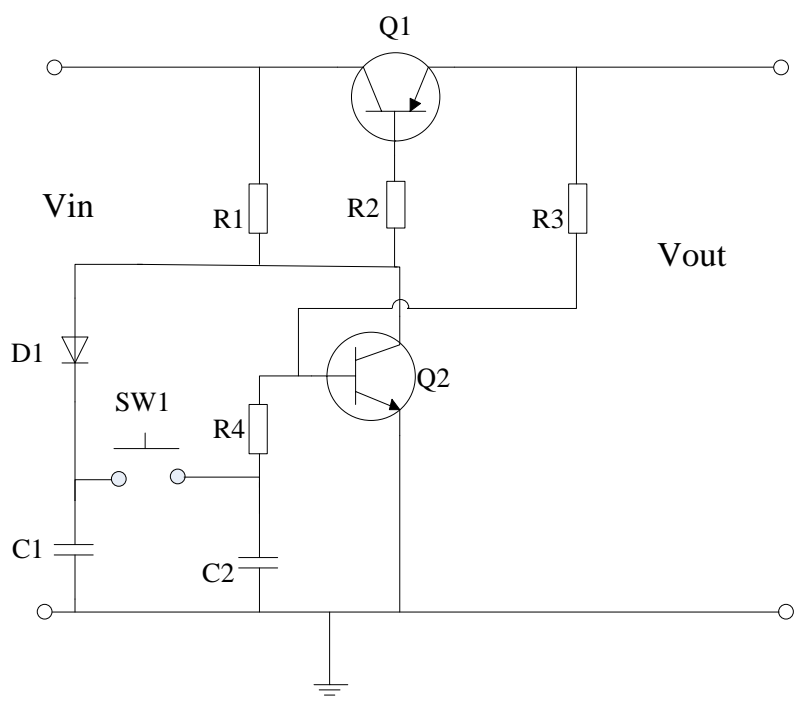

Fig.2: Light touch power switch based on transistors

If turning on the power is needed, press the button SW1 slightly. Because C1 is full of electronics, Q2 turns on thanks to the large bias current after press the button. D1 prevents the instantaneous impact of current on the input power. Q1 tube also conductes by the bias current and the output power is through the load. Q2 tube obtains bias current from the output terminal through R3, so as to further guide. Due to the positive feedback effect of Q2 and Q1 transistors in each other, the switch is quickly into a closed state in a very short period of time. After the Q2 transistor is turned on, the capacitor C 1 is discharged through the Q2 to near zero level, and is ready for the next disconnection of the switch.

Press the button switch SW1 to turn off the circuit when it is on. Because the voltage on the capacitor $\mathrm{C} 1$ is close to zero, Q2 turn into the instantaneous cut-off state and stop due to no bias current. The output voltage disappears quickly due to Q2 base without the bias current.While R3 can not provide current to Q2 base. Due to the negative feedback between Q2 and Q1, the switch will soon enter into the cut off state. After the SW1 has been released, it is the same as the status and process of the power supplied. C1 charging is ready for the next closed state.

\subsection{Light touch power supply switch based on triggers}

In addition to transistors, flip flops are also a good choice, taking the advantages of a clearer logic and easier to grasp the control process. With the development of the integrated circuit industry, the package size of the trigger is also close to the transistor, so there is a lot of space in the portable power switch. ${ }^{4}$

Base has no bias current. R3 can't provide current to Q2 base. Due to the negative feedback between Q2 and Q1, the switch will soon enter into the cut off state. After the SW1 has been released, it is the same as the status and process of the power on.

Common triggers include SR trigger, $\mathrm{T}$ trigger, $\mathrm{D}$ trigger and so on. All of them can be used as part of the digital switch in light touch type power supply switch. The following is an example of 74LVC1G74, the trigger as the realization of digital switch.

74LVC1G74 is a single D trigger with a start and reset function, rising edge triggered. The synchronization truth table is shown in Table 1, and the asynchronous truth table is shown in table 2.

\section{Please note:}

$\mathrm{H}=\mathrm{HIGH}$ voltage level;

$\mathrm{L}=\mathrm{LOW}$ voltage level;

$\mathrm{X}=$ don't care;

$\uparrow=$ LOW-to-HIGH CP transition;

$\mathrm{Qn}+1=$ state after the next LOW-to-HIGH CP transition

\begin{tabular}{|c|c|c|c|c|c|}
\hline \multicolumn{5}{|c|}{ INPUT } & \multicolumn{2}{c|}{ OUTPUT } \\
\hline$\overline{\mathbf{S}} \mathbf{D}$ & $\overline{\mathbf{R}} \mathbf{D}$ & $\mathbf{C P}$ & $\mathbf{D}$ & $\mathbf{Q}$ & $\overline{\mathbf{Q}}$ \\
\hline $\mathrm{L}$ & $\mathrm{H}$ & $\mathrm{X}$ & $\mathrm{X}$ & $\mathrm{H}$ & $\mathrm{L}$ \\
\hline $\mathrm{H}$ & $\mathrm{L}$ & $\mathrm{X}$ & $\mathrm{X}$ & $\mathrm{L}$ & $\mathrm{H}$ \\
\hline $\mathrm{L}$ & $\mathrm{L}$ & $\mathrm{X}$ & $\mathrm{X}$ & $\mathrm{H}$ & $\mathrm{H}$ \\
\hline
\end{tabular}

Table 1: Asynchronous operation

\begin{tabular}{|c|c|c|c|c|c|}
\hline \multicolumn{5}{|c|}{ INPUT } & \multicolumn{2}{c|}{ OUTPUT } \\
\hline$\overline{\mathbf{S}} \mathbf{D}$ & $\overline{\mathbf{R}} \mathbf{D}$ & $\mathbf{C P}$ & $\mathbf{D}$ & $\mathbf{Q}_{\mathbf{n}+1}$ & $\overline{\mathbf{Q}}_{\mathrm{n}+1}$ \\
\hline $\mathrm{H}$ & $\mathrm{H}$ & $\uparrow$ & $\mathrm{L}$ & $\mathrm{L}$ & $\mathrm{H}$ \\
\hline $\mathrm{H}$ & $\mathrm{H}$ & $\uparrow$ & $\mathrm{H}$ & $\mathrm{H}$ & $\mathrm{L}$ \\
\hline
\end{tabular}

Table 2 Synchronous operation 
The synchronous trigger state is equivalent to the level trigger. The state of the SD and RD determines the Q and Q non state, independent of clock CP and D. If there are two switches need to be controlled and only one way is connected, synchronous trigger logic is very suitable. But two logic states to control or the inverter will be needed. The synchronous trigger state is not suitable for touch switch, because light touch switch can not let the input level keep two constant states. But small mechanical switches are a good choice. Therefor, light touch power switch chooses in the asynchronous trigger mode, through the external trigger to control clock CP state and control the power switch system output state.

The circuit diagram using in this design is shown in figure 3. According to the truth table of the asynchronous mode, RD and SD are pulled high and D and Q are connected together. Each time the clock changes to the rising edge, the $\mathrm{Q}$ state becomes the current state of the $D$. The state of the $D$ is consistent with the current $\mathrm{Q}$ non - state. Each single trigger achieves the current state of the $\mathrm{Q}$ non - state down to the next phase of $\mathrm{Q}$. Once $\mathrm{CP}$ is triggered, the $\mathrm{Q}$ switches between high and low levels. Furthermore, the control of V1 grid is realized. V1 is a $\mathrm{N}$ enhanced field effect transistor and the V2 is a P enhanced field effect transistor. Large current can flow from the V2 and V1 only play the role of control.
In the initial state, the V2 gate is pulled up through the R4 and the V2 is turned off. The VOUT has no output. When the clock is pulled up to a high level and the C1 is charged to a high level. When the switch button SW is pressed, the 1 term of the switch button is quickly changed to a low level. However, due to the presence of $\mathrm{C} 1$, the trigger clock can not immediately respond. C1 discharges gradually until the low level. At this time, the $\mathrm{CP}$ is equivalent to the falling edge and the output of the flip-flop Q will not change. When the switch SW is released, the power supply charges the C1 until the high level VIN. For the clock CP, the rising edge is completed. The $\mathrm{Q}$ of the trigger will change at this time. In this process, C1 played a role in preventing misoperation. When the switch SW is pressed light, the CP can not complete the change in the rising edge. In addition, C1 can also control the slope of the rising edge of CP. According to the requirements of the flip-flop on the edge, $\mathrm{C} 1$ can choose a larger capacitor.

When the flip-flop Q output is high, the V1 is switched on. The gate of the V2 is pulled down to a low level, and the V2 is turned on to complete the input changing to the output power supply. The device is ready for the next state. When the power is turned on, if the button is pressed and released, the output of the flip-flop Q becomes low. V1 shut down and the V2 gate is pulled high, the V2 is turned off. The power switch system is realized and the device is turned off.

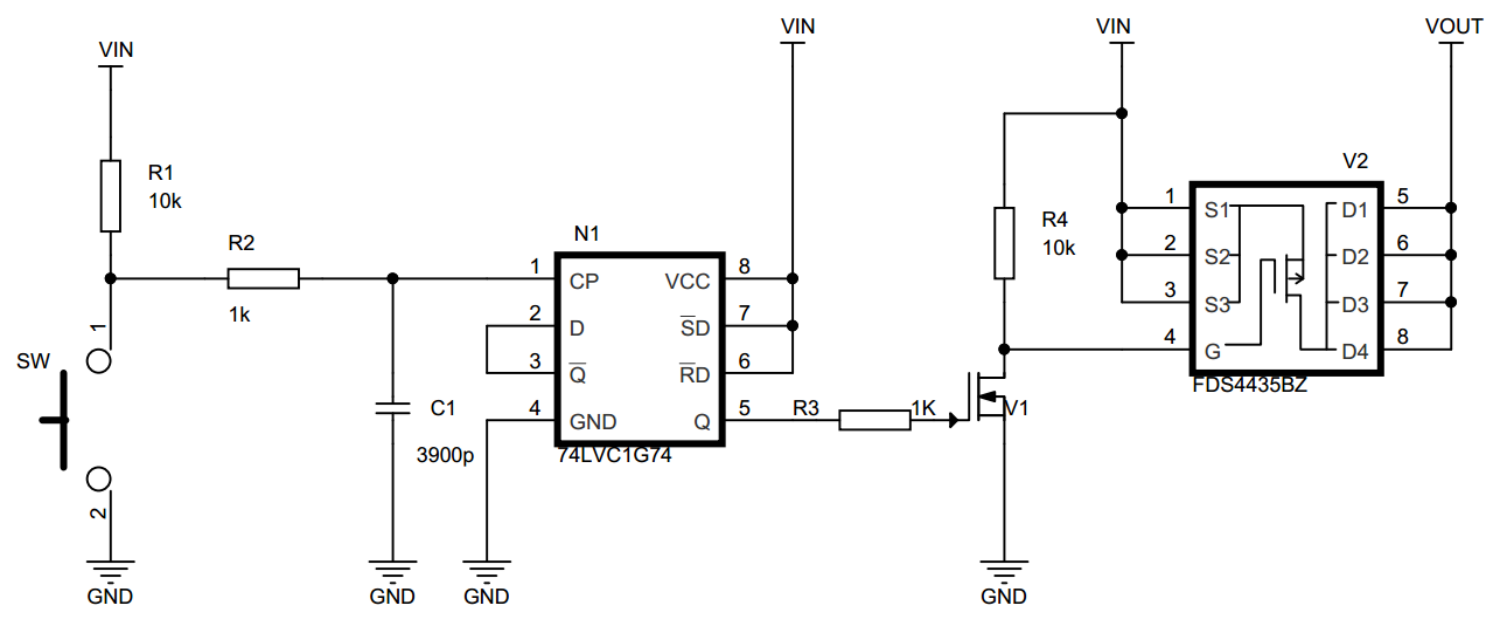

Fig.3: Light touch power supply switch based on triggers

\subsection{Light touch power switch based on microprocessor}

The transistor and trigger can control touch switch power good. But if you want to achieve some additional functions, the two circuits cannot be achieved. Microprocessor will be a good choice. The microprocessor can be chosen according to the complexity of the function. At the same time, more functions means that the cost will increase accordingly. However, the microprocessor which can realize the complex function is often larger in size, which does not meet the requirements of portable. This design uses the microprocessor, the volume is small, the price is cheap. The utility model can effectively realize the control of the switch system, the circuit structure is simpler, and can also provide some additional functions.

This case uses the STC15 class processor chip. This microcontroller is a localization of the device. Therefor, the localization of the requirements of the design can refer to. And the price is much lower than a single transistor or trigger, but also to reduce the cost of great benefit.

A brief introduction to the STC15 series chips, according to different needs can choose different supply voltage, support $3.3 \mathrm{~V}$ and $5 \mathrm{~V}$. The processor includes watchdog circuitry, 
counters, registers, etc. Burning program has a dedicated download, but also supports online baking, easy to change the internal procedures. In addition to 5 feet and 6 feet need to be reserved for online programming, 1, 3, 7, 8 feet can be used to receive and control. Greatly increase the freedom of control. If the power supply in addition to the switching requirements, but also the state indicator function, without additional devices, further effectively reduce the cost.

The initial state, the internal program to initialize the microcontroller 8 feet high, when the SW pin is low, the state of the 8 pin changes. The initial state of the microcontroller 7 feet is pulled high. Press the switch button SW, the 7 pin is placed low and the state of the 8 pin is changed to a low level. Transistor V1 is turned on, power output is complete.

When the power supply is turned on, press the switch button SW, the 7 pin is placed low, the state of the 8 pin is changed to a high level. Transistor V1 cuts off and the switch system powers off.

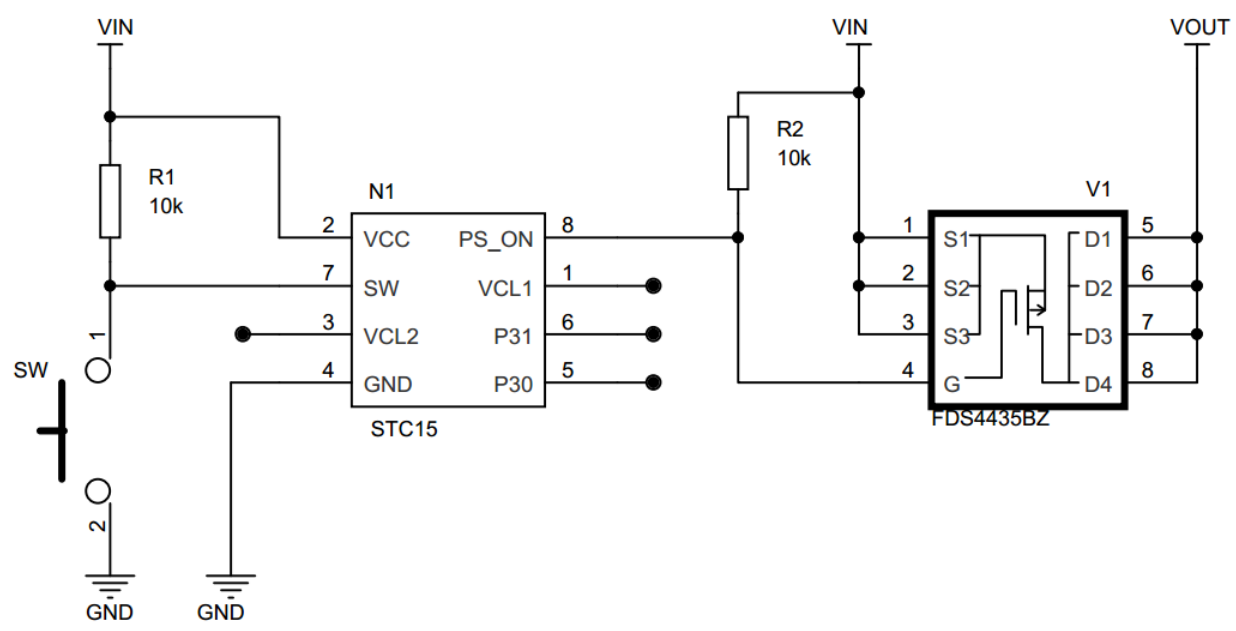

Fig.4: Light touch power supply switch based on microprocessor

\section{Conclusion}

Miniaturization, portable equipment is the trend. Therefore, the scope of application of touch type power supply switch will become more and more widely. Through the introduction of several examples in this paper, the author hopes to provide reference for engineering designers and researchers. When the complexity is not high, transistors and triggers should be preferred. In particular, the transistor structure mentioned in this paper can effectively shut off the self power supply. If additional features are needed, the microprocessor is a good choice. According to the requirements of the complexity and other requirements, select the appropriate microprocessor.

\section{Acknowledgements}

The author wishes to thank the funding organisations for providing this template and He peng previously provided technical support and assistances.

\section{References}

[1] Guilbert, Damien. "Investigation of the interactions between proton exchange membrane fuel cell and interleaved DC/DC boost converter in case of power switch faults.” international journal of hydrogen energy pp519-537 (2015)
[2]. Wang, Yanzhi, Xue Lin, and Massoud Pedram. “Optimal power switch design methodology for ultra dynamic voltage scaling with a limited number of power rails.” Proceedings of the 24th edition of the great lakes symposium on VLSI. ACM,(2014).

[3].Sato, Takashi, et al. "Grease for electrical contact and slide electricity structure, power switch, vacuum circuit breaker, vacuum insulated switchgear, and vacuuminsulated switchgear assembling method.” U.S. Patent No. 9.784,( 2016) .

[4]. S.A.Shirsavar. "Teaehing Praetieal Designing of Switch Mode Supply[J].” IEEE Transaetionson Edueation PP6063, (2004).

[5]. Tsai Cheng-hua, Tsai Jui-che. MEMS optical switches and interconnects[J].Displays,pp33-40(2015). 